\title{
Report on WHO AIFO Joint Workshop
}

A workshop entitled 'Working Together for the Consequences of Leprosy' was held at the World Health Organisation (WHO) in Geneva, 25-27 September 2000. The workshop was hosted by WHO and jointly supported by AIFO (Associazione Italiana Amici di Raoul Follereau). The participants included representatives of these two organizations as well as national governments, and national and international non-governmental organizations. This is a draft summary of the workshop and a full report of the workshop is being prepared for publication.

\section{Summary}

More than 10 million persons affected by leprosy have been cured with MDT in the last decade. However, many of those cured still continue to face problems due to existing or potential, physical and/or psycho-social disabilities. Many of them need particular care of the affected eyes, hands and feet to prevent onset of serious disabilities.

The main objective of the workshop was that of promoting a better understanding of the rehabilitation needs of persons having physical, psychological, and socio-economic disabilities due to leprosy. Another task was that of identifying strategies for leprosy work in the changing context of health services, leprosy control programmes, rehabilitation services, and community-based rehabilitation (CBR) programmes.

During the workshop, an attempt was made to quantify rehabilitation needs deriving from leprosy in specific areas or countries, as well as world-wide. Report about the global situation of leprosy as well as some country/project reports from Brazil, Ghana, Indonesia, China and Karnataka State (India), were presented. Based on global data from WHO, there are an estimated 3 million persons with leprosy-related physical disabilities (grade 1 and 2) in the world. Most of these need to practice prevention of impairment and disability (POID) activities to prevent worsening of existing impairments. Studies done by IDEA, DAHW and ALES suggest that up to $35 \%$ of leprosy-affected persons may need socio-economic rehabilitation.

Reports and discussions provided the basis for the identification of some key issues, for which the workshop participants made the recommendations. It was acknowledged that changes in rehabilitation approaches are required as a result of decreasing prevalence of disease and the shift from vertical leprosy programmes to the integration of leprosy into the PHC (Primary Health Care) system. New approaches would also help to decrease stigma and to increase coverage of POID and rehabilitation services.

Emphasis was placed on the need to adopt a multi-sectoral, holistic approach to address the issue of physical and socio-economic rehabilitation. The different and complex issues related to the rehabilitation of leprosy disabled persons can only be adequately addressed if 
the affected persons, their families, communities, leprosy experts, and authorities in various sectors act as true partners. Empowerment and active participation of affected persons were acknowledged as indispensable, particularly in identification of rehabilitation needs, advocacy and the creation of effective networks at various levels.

For better assessment of physical disabilities and rehabilitation needs, leprosy programmes should be encouraged to collect disability data on a cohort basis. Particular attention may be needed for countries which have reached the elimination goal and where incidence of disability among new cases is increasing.

Despite the encouraging achievements of the past decade, some of the old leprosaria may be still needed to take care of persons with severe chronic disabilities. 